\title{
EXPLOITING SATELLITE FOCAL PLANE GEOMETRY FOR AUTOMATIC EXTRACTION OF TRAFFIC FLOW FROM SINGLE OPTICAL SATELLITE IMAGERY
}

\author{
Thomas Krauß
}

DLR - German Aerospace Center, Remote Sensing Institute, Münchener Str. 20, 82234 Oberpfaffenhofen, thomas.krauss@dlr.de

\author{
Commission I, WG I/4
}

KEY WORDS: Optical Satellite Data, Focal plane assembly, Traffic detection, Moving objects detection

\begin{abstract}
:
The focal plane assembly of most pushbroom scanner satellites is built up in a way that different multispectral or multispectral and panchromatic bands are not all acquired exactly at the same time. This effect is due to offsets of some millimeters of the CCD-lines in the focal plane. Exploiting this special configuration allows the detection of objects moving during this small time span. In this paper we present a method for automatic detection and extraction of moving objects - mainly traffic - from single very high resolution optical satellite imagery of different sensors. The sensors investigated are WorldView-2, RapidEye, Pléiades and also the new SkyBox satellites.
\end{abstract}

Different sensors require different approaches for detecting moving objects. Since the objects are mapped on different positions only in different spectral bands also the change of spectral properties have to be taken into account. In case the main distance in the focal plane is between the multispectral and the panchromatic CCD-line like for Pléiades an approach for weighted integration to receive mostly identical images is investigated. Other approaches for RapidEye and WorldView-2 are also shown. From these intermediate bands difference images are calculated and a method for detecting the moving objects from these difference images is proposed.

Based on these presented methods images from different sensors are processed and the results are assessed for detection quality - how many moving objects can be detected, how many are missed - and accuracy - how accurate is the derived speed and size of the objects. Finally the results are discussed and an outlook for possible improvements towards operational processing is presented.

\section{INTRODUCTION}

Actual very high resolution (VHR) satellite sensors are mostly operated as pushbroom scanners with different CCD-arrays for each panchromatic or multispectral band. If these arrays are mounted in distances of millimeters or even centimeters in the focal plane assembly (FPA) the same ground point is not acquired at the same time in all CCD-arrays. This principle is shown in fig. 1.

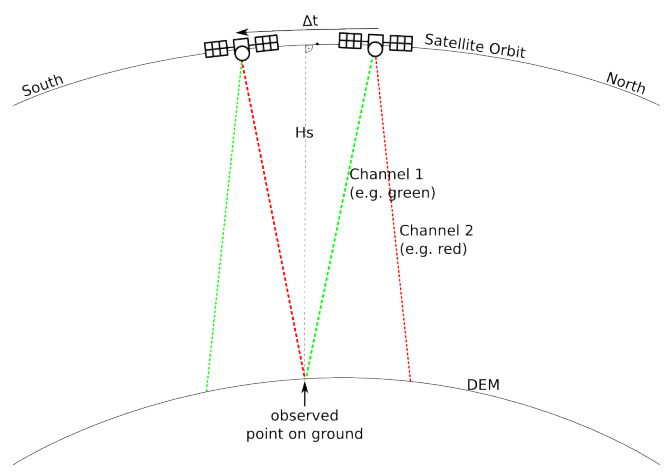

Figure 1: Principle of acquisition geometry of image bands separated in a FPA

If moving objects are recorded by a sensor whith such an acquisition system the object appears in different positions at each band. See for an example fig. 2. This image shows a part of an RapidEye satellite scene containing a plane. The plane appears at different positions in the blue, green and red band.

In this work we will exploit this feature of many sensors to automatically detect moving traffic in satellite images. The sensors

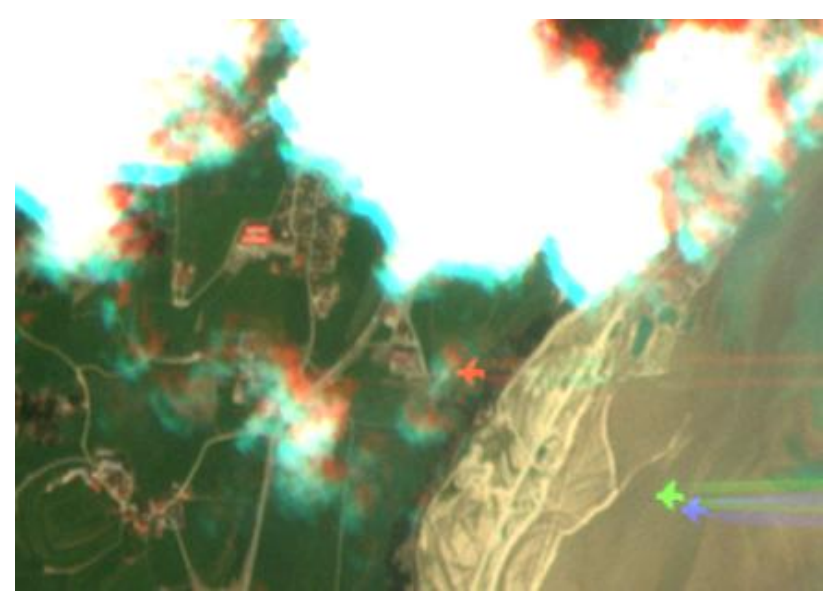

Figure 2: Section $2.1 \times 1.5 \mathrm{~km}$ from a RapidEye scene of southern bavaria (north of Füssen) containing clouds and a plane

investigated are WorldView-2, RapidEye, Pléiades and also the new SkyBox satellites.

These sensors are like most of all very high resolution earth observation sensors built up as pushbroom scanners or a special pushbroom-frame-camera configuration in the case of SkyBox. To acquire different spectral bands for each band one CCD line is necessary. Most sensors have the panchromatic CCD line and the multispectral CCD scan lines mounted separately on the focal plane assembly with a distance of several millimeters. Others - like RapidEye or WorldView-2 - have even different multispectral CCD scan lines mounted separately. In the case of 
RapidEye there is a large gap in the size of several millimetres between the scan lines for red/red-edge/near-infrared (NIR) and green/blue but only about 6.5 micrometres inside the lines between e.g. green and blue. This assembly results in the colored cyan/red corners which can be detected easily in each RapidEye image containing clouds (see also fig. 2 for this effect). For WorldView-2 there exist two four-line multispectral CCD lines one for the "classic" bands blue/green/red/NIR and one for extra bands coastal/yellow/red-edge/NIR2. Between these two fourchannel-CCD-lines the panchromatic CCD-line is located. For Pléiades there exist one multispectral four-channel-CCD-line and one panchromatic CCD-line. In case of the SkyBox satellites the configuration is a little more complicated. SkyBox uses three frame sensors, each divided in a panchomatic part in the upper half and the four multispectral bands blue/green/red/NIR in the lower half of the frame. Operated in the scanning mode there is also a small time distance between each of the color bands and also to the panchromatic band.

First we present the design of the focal plane assembly (FPA) of each of the sensors to describe which bands are selected for the moving object detection. Second we describe the method for automatically extraction of moving objects. Afterwards the method is applied to images of the different sensors and the results are shown and evaluated. Finally the method is assessed and an outlook for further improvements of the method is given.

But first let's have a look on the focal plane assemblies, acquisition principles and example imagery of our sensors.

\section{ACQUISITION PRINCIPLES AND FOCAL PLANE ASSEMBLIES}

\subsection{WorldView-2}

The WorldView-2 multispectral instrument consists of two multispectral CCD lines acquiring in the first the standard channels blue, green, red and the first near infrared band and in the second the extended channels coastal blue, yellow, red edge and the second near infrared band. These two CCD lines are mounted on each side of the panchromatic CCD line. Therefore the same point on ground is acquired by each line at a different time. Fig. 3 shows the focal plane assembly (FPA) of WorldView-2.

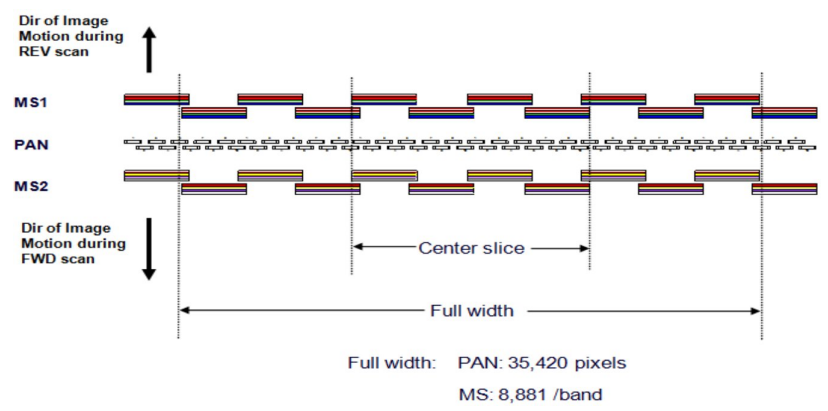

Figure 3: Focal plane assemblies of WorldView-2 (sketch courtesy Digital Globe)

In table 1 from Kääb (2011) the time lags for the sensor bands are given.

In our investigations we use the yellow and red bands from MS2 and MS1 respectively due to the good spectral correlation for most traffic objects in this spectral range. The time difference $\Delta t_{W V 2}$ for these two bands corresponding to table 1 is $0.340 \mathrm{~s}-$ $0.016 \mathrm{~s}=0.324 \mathrm{~s}$ which is in good correlation to our calibration results of $\Delta t_{y r}=0.297 \pm 0.085 \mathrm{~s}$ as derived in Krauß et al.
Table 1: WorldView-2's recording properties

\begin{tabular}{|l|l|l|l|l|}
\hline $\begin{array}{l}\text { Band recording } \\
\text { data order }\end{array}$ & $\begin{array}{l}\text { Sensor } \\
\text { name }\end{array}$ & $\begin{array}{l}\text { Wavelength } \\
{[\mathrm{nm}]}\end{array}$ & $\begin{array}{l}\text { Inter-band } \\
\text { Time lag }[\mathrm{s}]\end{array}$ & $\begin{array}{l}\text { Time lag }[\mathrm{s}] \\
\text { from start }\end{array}$ \\
\hline Near-IR2 & MS2 & $860-1040$ & Recording start & Recording start \\
Coastal Blue & MS2 & $400-450$ & 0.008 & 0.008 \\
Yellow & MS2 & $585-625$ & 0.008 & 0.016 \\
Red-Edge & MS2 & $705-745$ & 0.008 & 0.024 \\
\hline Panchromatic & PAN & $450-800$ & & \\
\hline Blue & MS1 & $450-510$ & 0.3 & 0.324 \\
Green & MS1 & $510-580$ & 0.008 & 0.332 \\
Red & MS1 & $630-690$ & 0.008 & 0.340 \\
Near-IR1 & MS1 & $770-895$ & 0.008 & 0.348 \\
\hline
\end{tabular}

(2013). Fig. 4 shows a section $(800 \times 400 \mathrm{~m})$ of a WorldView- 2 scene in the north of Munich (A99) consisting of the yellow and red band.

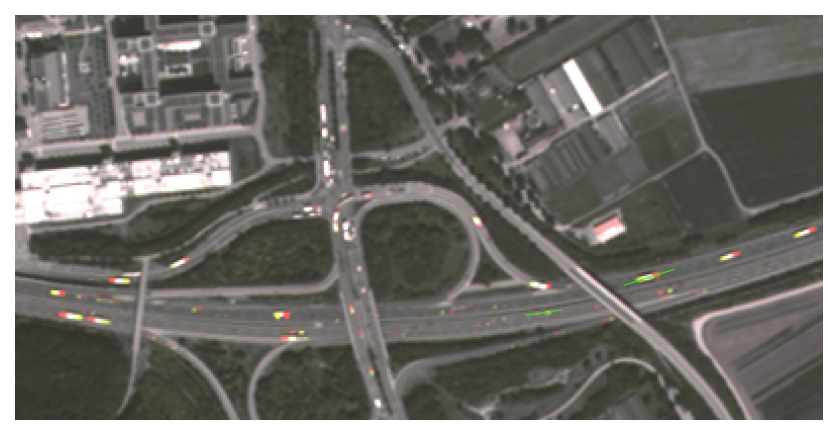

Figure 4: Displacement of cars seen by WorldView-2 in the red and yellow band (section $800 \times 400 \mathrm{~m}$ on A99 north of Munich)

In this image the displacement of moving cars in the two channels is clearly visible. Knowing the right-hand-traffic in Germany we see, the red band is acquired earlier than the yellow band and the image was acquired in forward direction. Fig. 5 shows the two profiles of a car (left, also left green profile-line in fig. 4) and a large truck (right).
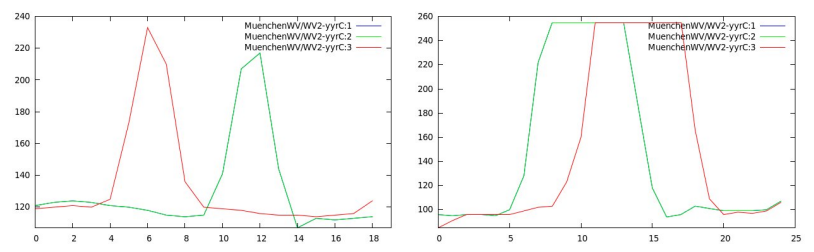

Figure 5: Profiles of a car (left) and a truck (right); red channel shown in red, yellow channel in green

\subsection{RapidEye}

As shown in fig. 6 the RapidEye focal plane assembly consists of five separate CCD lines - one for each band. They are grouped in two mounts: the blue and green on one and the red, red edge and the near infrared band on the second.

So the main gap can be found between the blue/green and the $\mathrm{red} / \mathrm{RE} / \mathrm{NIR}$ bands. As calibrated in Krauß et al. (2013) the time gap between the red and the green band is $\Delta t_{r g}=2.65 \pm 0.50 \mathrm{~s}$. Fig. 2 shows a typical RapidEye image containing clouds and a crossing plane. In fig. 7 a section of the highway A96 near Inning in Germany is shown. The cars can be only vaguely detected as blurred red and cyan blobs on the highway. From this figure already the challenge can be imagined to detect such objects automatically. 


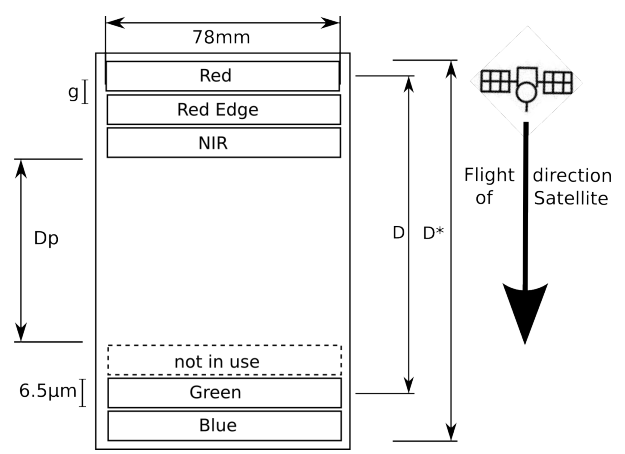

Figure 6: RapidEye focal plane assembly (FPA), $g$ : gap between lines, $D_{p}$ : distance between packages, $D^{*}$ : maximum distance between lines, $D$ : distance red-green

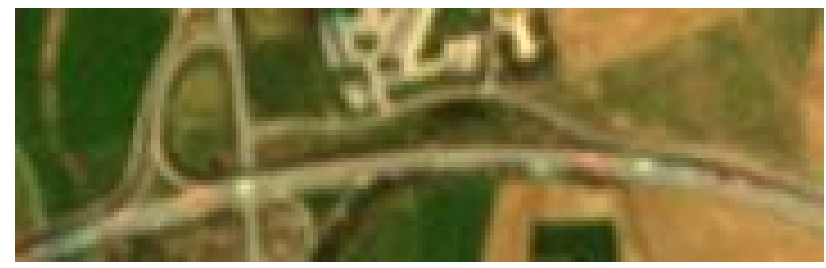

Figure 7: Displacement of cars seen by RapidEye (in cyan/red, section $900 \times 280 \mathrm{~m})$

\subsection{Pléiades}

The Pléiades FPA is similar but consists only of one multispectral and one panchromatic sensor line. The main gap exists only between the multispectral bands and the pan channel where the latter is also mounted in a curvature around the optical distortion center (marked with $\mathrm{a} \times$ in the figure) as shown in fig. 8 . Delvit et al. (2012) stated for the time difference between the multispectral and the PAN CCD $\Delta t_{m s, p a n}=0.15 \mathrm{~s}$ where we found in our previous calibration (Krauß et al., 2013) a value of $\Delta t_{m s, p a n}=0.16 \pm 0.06 \mathrm{~s}$.

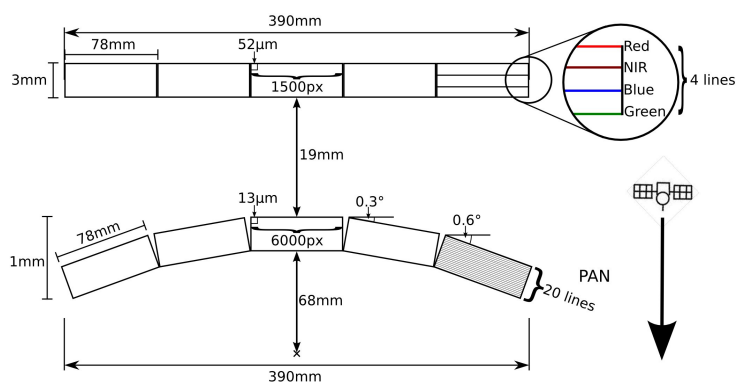

Figure 8: Focal plane assembly Pléiades (curvature of PAN sensor strongly exaggerated)

In fig. 9 a section $160 \times 100 \mathrm{~m}$ of the M1 in Melbourne near the harbour is shown. The PAN channel in red, the combined multispectral channels in cyan. Since in Australia is left handed traffic the cars are travelling from left to right. So the PAN channel is acquired before the multispectral bands. In fig. 10 the two profiles along the green lines in fig. 9 are shown. The left profile is the profile of the truck (left in fig. 9), the right profile the profile of the two cars on the right.

To combine the PAN and multispectral channels for a parallel processing the PAN channel has to be reduced in resolution and the multispectral bands have to be combined to a panchromatic band.

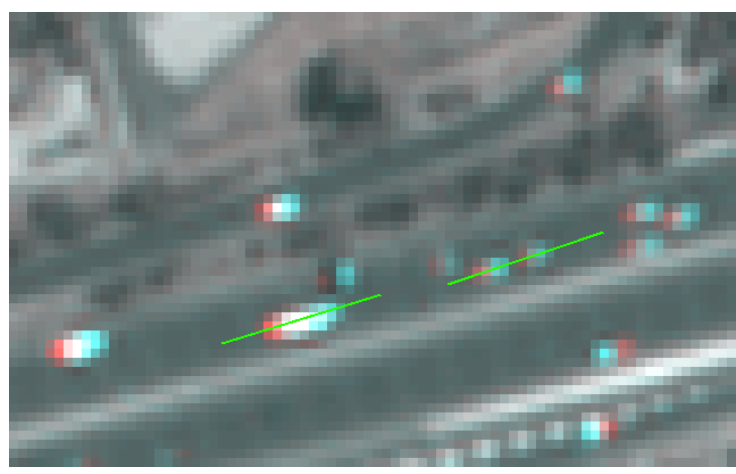

Figure 9: Example of a combined PAN (red) and multispectral (cyan) Pléiades image, section $160 \times 100 \mathrm{~m}$ of the M1 in Melbourne near the harbour.
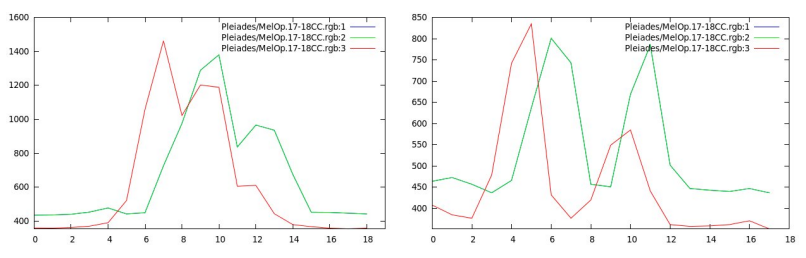

Figure 10: Profiles along the green lines from fig. 9, left: truck (also left in image), right: two cars, all travelling from left to right, $\mathrm{DN}$ vs. metres

Using the spectral response function of the multispectral bands (weights $w_{i}$ corresponding to the part of the multispectral band contained in PAN channel) and taking into account the physical gains $g_{i}$ as listed in tab. 2 a synthetic panchromatic band can be calculated from the multispectral bands $M S_{i}$ as follows:

$$
P_{m s}=\frac{\sum_{i=1}^{4} w_{i} \cdot M S_{i} \cdot g_{i}}{g_{p} \cdot \sum_{i=1}^{4} w_{i}}
$$

Table 2: Weights $w$ and gains $g$ for the investigated Pléiades scene

\begin{tabular}{|l|l|l|l|}
\hline Index & Band & Weight $w$ & Gain $g$ \\
\hline 1 & Blue & 0.3 & 0.104275286757039 \\
2 & Green & 1.0 & 0.109170305676856 \\
3 & Red & 0.9 & 0.0968054211035818 \\
4 & NIR & 0.5 & 0.0639386189258312 \\
\hline$p$ & PAN & 2.7 & 0.0880281690140845 \\
\hline
\end{tabular}

On the other hand the PAN channel has to be resampled correctly to the 4-times lower resolution multispectral bands. This can be achieved by scaling down the PAN channel using an area averaging and applying a gaussian filter with $\sigma=0.7 \mathrm{px}$ :

$$
P_{\text {pan }}=\gamma_{0.7}\left(\operatorname{scale}_{1 / 4}(P A N)\right)
$$

\subsection{SkyBox}

The SkyBox satellites carry three frame cameras as shown in fig. 11. These cameras acquire overlapping images for a whole strip. The overlap of the images is about $97 \%$. This means each pixel of the PAN channel will be combined from about 20 images and each pixel of each of the multispectral bands will be combined from 4 frame camera images. 


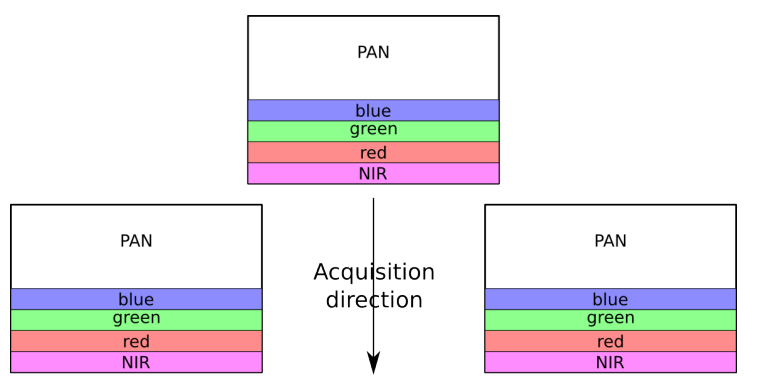

Figure 11: Focal plane assembly SkyBox, three frame cameras splitted in a PAN and four multispectral bands

Fig. 12 shows an example of a plane crossing the acquisition path of SkyBox. The image is a orthorectified composite of the 1-mPAN-band (in gray) and overlayed the four 2.5-m-multispectral bands (blue, green, red and NIR shown as purple). As can easily be seen in the PAN band the image consists of about 20 single frame camera images which are merged to one master image in the SkyBox level-1B-preprocessing. The same procedure is applied to the four half-resolution multispectral bands. But with the lower resolution no single planes will be visible here any more but only one blurred combined image. The distances of the centres of the plane images correlate with the FPA as shown in fig. 11.

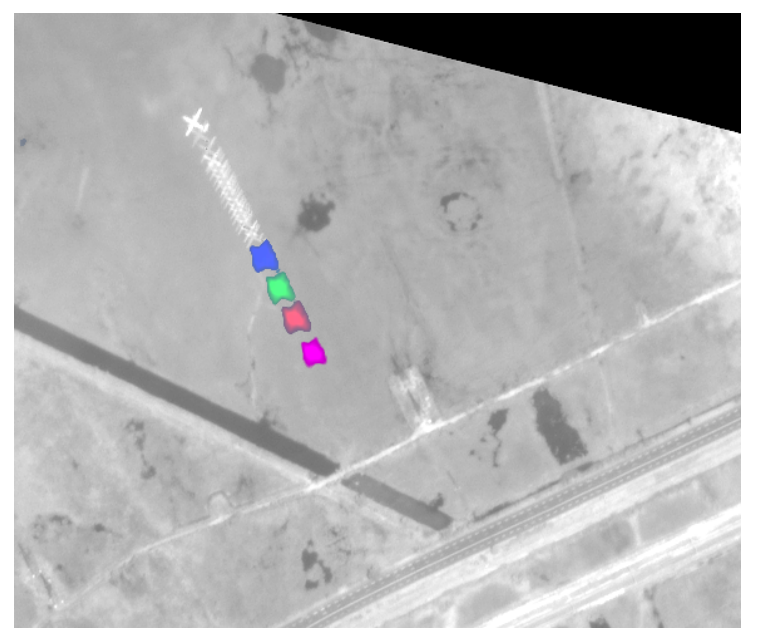

Figure 12: Example of a Skybox image showing a moving plane in PAN, blue, green, red and NIR channel (2014-08-09, detector 3 , image 6)

Fig. 13 shows a section of the N568 near Fos-sur-Mer (Camargue, France). Fig. 14 shows the two profiles marked in fig. 13, left the top, bright, right the lower, darker car.

The profiles are the $2.5 \mathrm{~m}$ multispectral image data resampled to the 1-m-PAN ortho-image. As can be seen, the cars - merged from four camera-frames - are shown as smooth curves. The maxima of each curve can be correlated to estimate the speed.

For SkyBox no calibration of the time gap $\Delta t$ exists until now. But from the NORAD two line elements (TLE) we can derive an average height of $h_{s}=581.5 \mathrm{~km}$ above earth and an average speed of $v=7564.25 \mathrm{~m} / \mathrm{s}$. This corresponds to an average ground speed of

$$
v_{g}=\frac{v \cdot R_{E}}{R_{E}+h_{s}}
$$

with an earth radius of $6371 \mathrm{~km} v_{g}$ become $6931.58 \mathrm{~m} / \mathrm{s}$. An original multispectral image with a nominal GSD of $2.39 \mathrm{~m}$ has

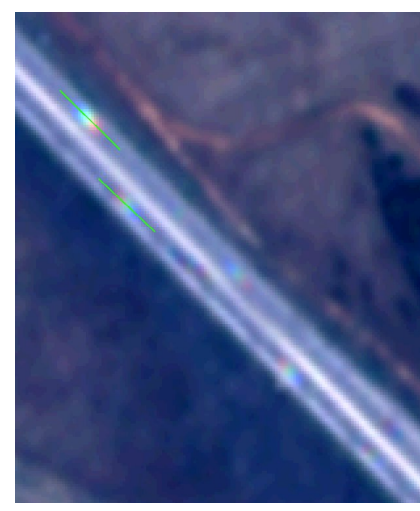

Figure 13: Skybox image showing the displacement of cars in the red, green and blue band, section $620 \times 750 \mathrm{~m}$, N568 near Fos-sur-Mer (Camargue, France)

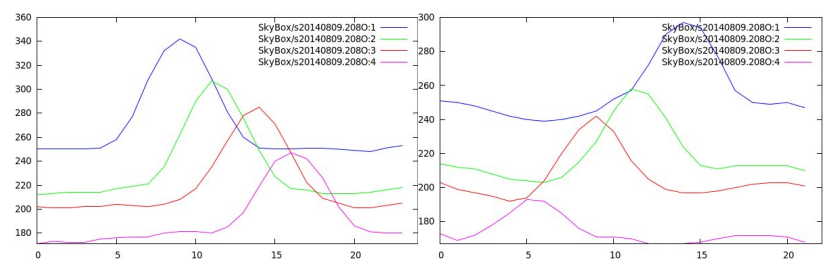

Figure 14: Profiles of two cars, left: top profile, right: lower profile from fig. 13, bands blue, green, red, NIR (as purple), digitial numbers (DN) vs. metres

540 rows or a length in flight direction of $l=1290.6 \mathrm{~m}$. So the acquisition of this image needs $t_{m s}=l / v_{g}=0.186 \mathrm{~s}$.

\subsection{Preliminary work}

In a previous investigation (Krauß et al., 2013) we showed how to calibrate the time gaps $\Delta t$ between different bands in single RapidEye and (multi-)stereo WorldView-2 and Pléiades images. The work was inspired in the detection of colored artifacts near moving objects.

Deeper analysis shows that this effect was already known from the first very high resolution (VHR) commercial satellites such as QuickBird and Ikonos. Etaya et al. (2004) uses already in 2004 QuickBird images of $0.6 \mathrm{~m}$ GSD panchromatic and $2.4 \mathrm{~m}$ multispectral and found a time gap between these bands of about $0.2 \mathrm{~s}$. In the same way M. Pesaresi (2007) found also a time lag of 0.2 seconds between the panchromatic and the multispectral bands of QuickBird images.

In an IGARSS paper Tao and Yu (2011) proposed the usage of WorldView-2 imagery for tracking moving objects. He calculated from a plane arriving at the Shanghai airport a time delay between the Coastal Blue Band on the second multispectral sensor line and the Blue Band on the first multispectral sensor line of about $17.5 \mathrm{~m} / 80 \mathrm{~m} / \mathrm{s}=0.216$ seconds.

Delvit (Delvit et al., 2012) described in his work on "Attitude Assessment using Pleiades HR Capabilities" the Pléiades focal plane (as shown in fig. 8). Here the panchromatic/multispectral shift is significant: $19 \mathrm{~mm}$ in the focal plane, which means $1 \mathrm{~km}$ on ground or a time delay of 0.15 seconds or in turn also a $1.5 \mathrm{mrad}$ stereoscopic angle. He also describes the maximum offset between two multispectral bands as 6 times smaller (maximum $3 \mathrm{~mm}$ ). The $1.5 \mathrm{mrad}$ stereoscopic angle means a height of about $300 \mathrm{~m}$ corresponds to $0.5 \mathrm{~m}$ shift (1 GSD of the pan channel). In turn 
using a matching accuracy of about 0.1 pixels allow for the extraction of a DEM with an uncertainty of $120 \mathrm{~m}(0.1 \times 4 \times 300 \mathrm{~m}$ for the multispectral GSD pixel size).

Also Leitloff (2011) gives in his PHD thesis a short overview of more of these methods and proposed also some approaches for automatic vehicle extraction of still traffic.

But none of these investigations tried to do an automatic detection of traffic in whole very high resolution (VHR) satellite scenes. All of the previous researches show only the possibility and derive the time gaps. In our here presented work we propose different methods tailored for the different sensors investigated to detect automatically some of the traffic in the imagery to derive traffic parameters like an average speed per road segment.

\section{METHOD}

As shown in the previous chapters all of the very high resolution (VHR) satellite sensors investigated in this paper allow the extraction of moving objects from only one single satellite image. This can be achieved by exploiting a small time gap $\Delta t$ in the acquisition of different bands as summarized in tab. 3 .

Table 3: Overview of time gaps and bands used for the investigated sensors

\begin{tabular}{|l|l|l|l|}
\hline Sensor & Bands & $\Delta t[\mathrm{~s}]$ & Resolution/GSD $[\mathrm{m}]$ \\
\hline WorldView-2 & yellow-red & 0.324 & 2.0 \\
RapidEye & green-red & $2.65 \pm 0.50$ & 5.0 \\
Pléiades & MS-PAN & $0.16 \pm 0.06$ & 2.0 \\
SkyBox & green-red & 0.186 & 2.4 \\
\hline
\end{tabular}

To correlate the needed bands they must have the same ground sampling distance (GSD) and should have the best possible similar spectral properties for the investigated objects. For traffic - cars and trucks - the red band and the nearest possible band with lower wavelength appeared to give the best correlations. So mostly in tab. 3 a red band together with a green or yellow band occur. The main exception is the Pléiades system where we have to create two synthetic low resolution panchromatic bands as explained in eqs. 1 and 2.

To detect the moving objects in a first step difference images between the above mentioned bands are created as shown in fig. 4. Fig. 15 shows the first step of the method where the bands involved are subtracted and the difference is median filtered with a merely large radius of about $18 \mathrm{~m}$ ( 9 pixels in the case of WorldView-2).

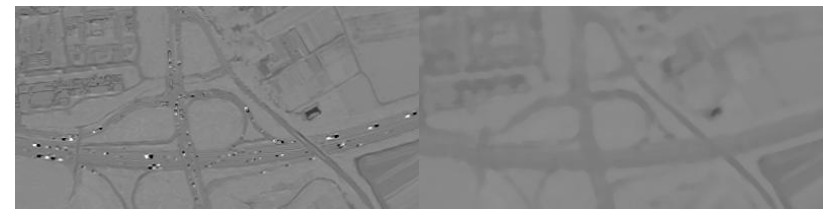

Figure 15: Sample processing of bands, step 1, example WorldView-2, left: difference image of red and yellow band, right: median filtered difference

Fig. 16 shows the second step of the object detection. Here the calculated median is subtracted from the difference image to emphasize only small local differences. Afterwards these differences are thresholded (1/6 of absolute brightness) and marked as positive or negative objects.

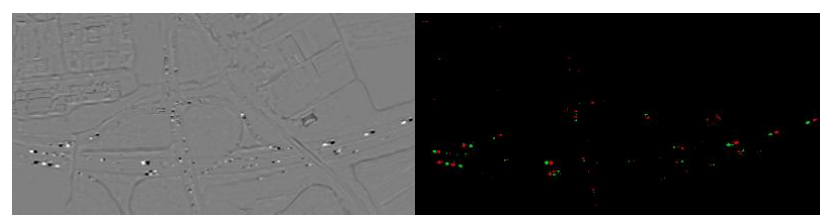

Figure 16: Sample processing of bands, step 2, example WorldView-2, left: difference image relative to median, right: detected positive and negative objects

In the third step the detected objects from fig. 16 (right) are fetched from the image and the nearest, best fitting (in sum of brightnesses) objects are taken as the "from" and "to" car positions. Using the distance of the centers of gravity of these positions together with the time gap $\Delta t$ for the bands of the sensor gives the speed of the object. Until now no restictions to road directions is included. So also a best match can be found across lanes.

\section{EXPERIMENTS}

\subsection{WorldView-2}

First we applied our method to a WorldView-2 dataset acquired on 2012-07-10 over Munich (Germany). Our method found 3615 objects in the whole area of $16.4 \times 19.8 \mathrm{~km}^{2}-$ mostly cars and trucks - in about 2 minutes. Two references where created manually: one containing all moving cars and trucks on highways and main roads as shown in fig. 17 (left, in red) and one containing all other moving traffic on all other roads in the image (left, in purple). Fig. 17 (right) shows all automatically detected objects overlaid in green.

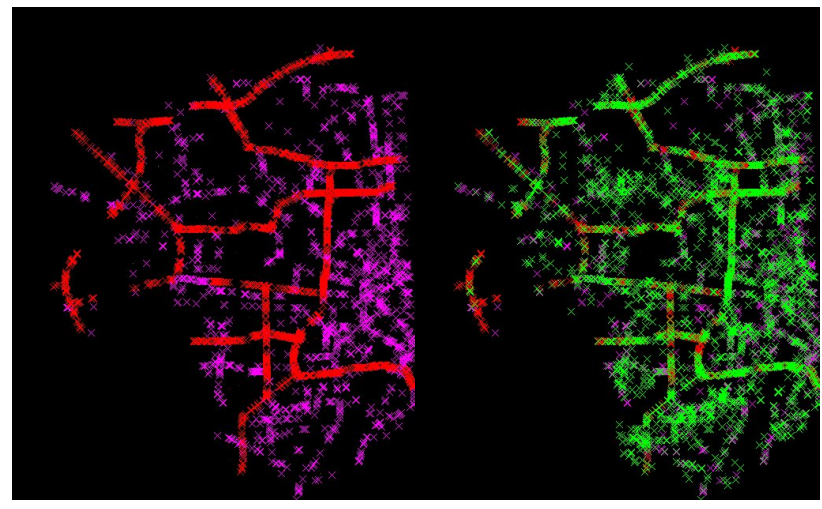

Figure 17: WorldView-2 scene, western half of Munich $(16.4 \times$ $19.8 \mathrm{~km}^{2}$ ), left: manually measured reference (cars on highways/main roads in red, all other cars in purple), right: automatically detected cars overlaid in green

In table 4 the results for the 3615 found objects against the manually measured objects are listed. In total 2063 objects from 4020 reference objects or $51 \%$ were detected correctly. But also a high rate of 1957 objects (49\%) was not detected. The wrongly detected 1552 objects ( $43 \%$ of all detected objects) can partly also be explained by missing objects in the manually measurement.

Typical objects which are not found by the proposed method have a too low contrast relative to the road. So the difference images show no strong signal on these positions and the objects cannot be detected. Also objects below thin clouds or haze are (partly) contained in the manual measurement but can not be found by the automatic method. Analysing on the other hand the false detected 
Table 4: Overview of time gaps and bands used for the investigated sensors

\begin{tabular}{|l|l|l|l|l|}
\hline Reference & $\begin{array}{l}\text { Objects } \\
\text { in reference }\end{array}$ & $\begin{array}{l}\text { Detected } \\
\text { correctly }\end{array}$ & $\begin{array}{l}\text { Detected } \\
\text { false }\end{array}$ & $\begin{array}{l}\text { Not } \\
\text { detected }\end{array}$ \\
\hline All & 4020 & 2063 & 1552 & 1957 \\
Highways/main roads & 2312 & 1083 & 2532 & 1229 \\
Other roads & 1708 & 997 & 2618 & 711 \\
\hline
\end{tabular}

objects show that some objects where detected by the automatic method which are existing but were just overseen by the manual measurements of about 6 students over 3 months.

Also a comparison of the detected speeds of the 3615 found objects was performed. Therefore for 2312 cars from the above mentioned reference both positions in the yellow and red band were measured. Fig. 18 shows the difference object image with the found derived speeds as green arrows and the original yellow/red bands with the manually measured objects as green crosses.

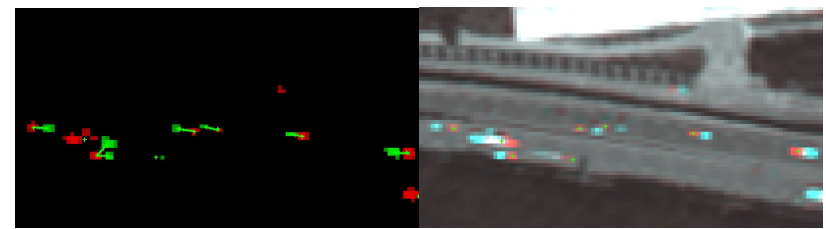

Figure 18: Correlation of measured vs. automatically detected speeds, left: difference object image, right: yellow/red image (in red/cyan), green crosses are the manual measurements of the from/to objects, the green arrows in the left image are the automatically derived speeds

The correlation of the automatically detected objects to these measured speed reference was done by taking a correlation if both positions (in the red and yellow band) of the detected and the manually measured object lied inside a correlation radius $\mathrm{rad}$ of 3,10 or 100 pixels.

Table 5: Accuracy assessment of detected speeds in the WorldView-2 scene

\begin{tabular}{|r|r|r|r|r|r|r|}
\hline rad & $\begin{array}{r}\text { Detected } \\
\text { correctly }\end{array}$ & $\begin{array}{r}\text { Reversed } \\
\text { direction }\end{array}$ & $\begin{array}{r}\text { Not } \\
\text { detected }\end{array}$ & $\begin{array}{r}\text { Detected } \\
\text { false }\end{array}$ & $\begin{array}{r}\text { Mean speed } \\
\bar{v}[\mathrm{~km} / \mathrm{h}]\end{array}$ & $\begin{array}{r}\text { Std. dev. } \\
\sigma_{v}[\mathrm{~km} / \mathrm{h}]\end{array}$ \\
\hline 3 & 894 & 50 & 1418 & 2721 & 13.1974 & 24.4084 \\
10 & 1067 & 238 & 1245 & 2548 & 24.5571 & 45.2691 \\
30 & 1187 & 425 & 1125 & 2428 & 25.6629 & 46.7234 \\
100 & 1377 & 707 & 935 & 2238 & 27.0640 & 49.1532 \\
\hline
\end{tabular}

Table 5 shows the calculated results of the correlation of the automatically detected to the manually measured objects together with the mean difference and standard deviation of the derived speeds. As can be seen more objects can be correlated between the automatic detected results and the manual measurement as the correlation radius increases. But also the mean speed difference and the standard deviation rises abruptly at a correlation radius higher than three pixels.

In fig. 19 the correlation of the detected objects is plotted for three correlation radii. As can be seen the blue points (correlation radius 3) fit already very good to the measurements. But between 50 and $100 \mathrm{~km} / \mathrm{h}$ there can be found a bunch of outliers where the automatically derived speeds lie between 150 and $200 \mathrm{~km} / \mathrm{h}$. This is due to the missing correct detection of trucks in the images. Please refer for the explanation to the profiles shown in fig. 5 referring to the yellow-red-image in fig. 4 .

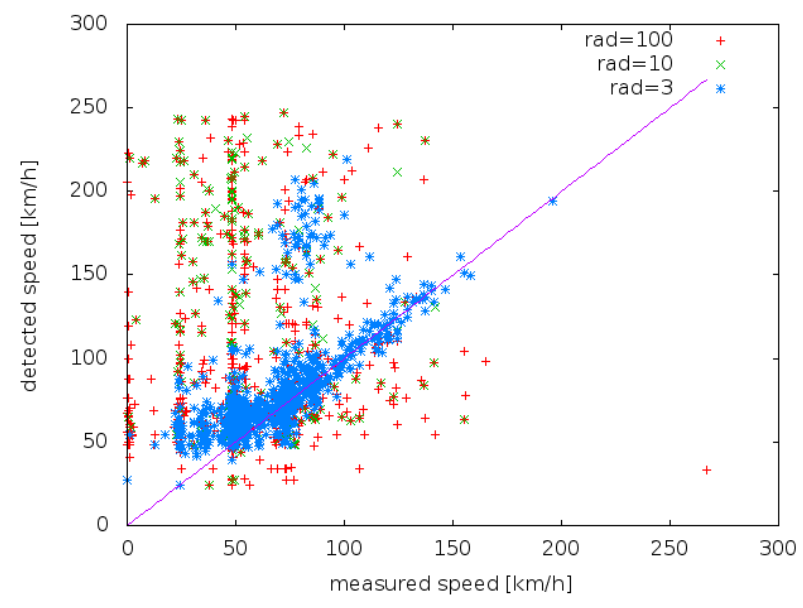

Figure 19: Correlation of measured vs. automatically detected speeds (correlation radius rad in pixels of GSD $2 \mathrm{~m}$ )

The method just does a difference of the image values. In the left case of fig. 5 (a car) there remains a red and a yellow blob which are correlated correctly. But in the case of a truck (right profile) the difference splits up the object to a left and a right object since the center part of the truck vanishes in the difference. In this case correlating the two split up blobs of the truck gives a virtual speed depending on the real speed and the length of the truck. To solve this problem the method has to be expanded to detect trucks as continous objects before doing the correlation.

\subsection{RapidEye}

For assessing the method with a RapidEye scene we use a scene acquired 2011-04-08 from an area west of Munich containing the A96 and the lakes Ammersee and Starnberger See as shown in fig. 20. The results for a small section $6 \times 2 \mathrm{~km}^{2}$ with the A96 near Schöffelding are shown in fig. 21.

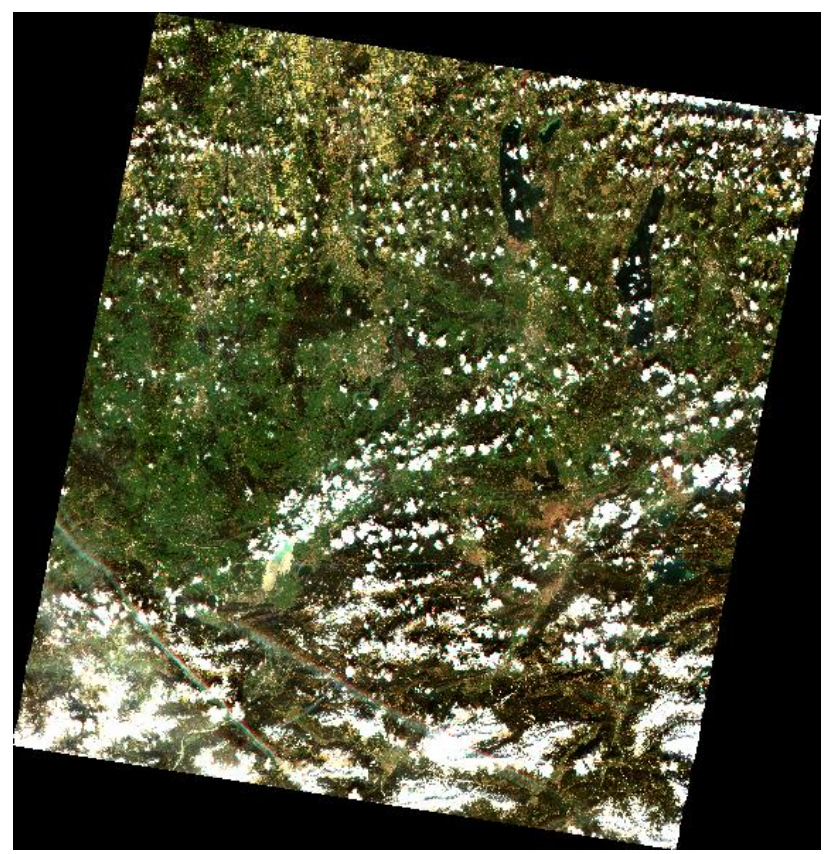

Figure 20: RapidEye Scene used for assesment, west of Munich, $100 \times 100 \mathrm{~km}^{2}$

In fig. 21 the correctly found cars on the highway A96 are marked with green arrows from the position in the first acquired green to the position in the red channel. In the center two cars can be seen 
driving with the same speed. A profile across these cars is shown in fig. 22.

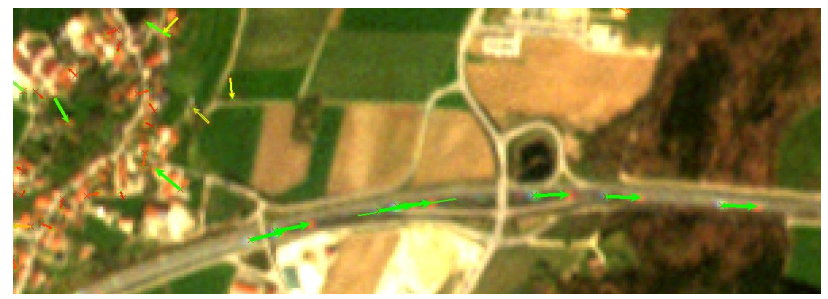

Figure 21: Objects found in part RapidEye Scene, $6 \times 2 \mathrm{~km}^{2}$, A96 near Schöffelding, green arrows denote movement of objects, green line across cars in center for profile in fig. 22

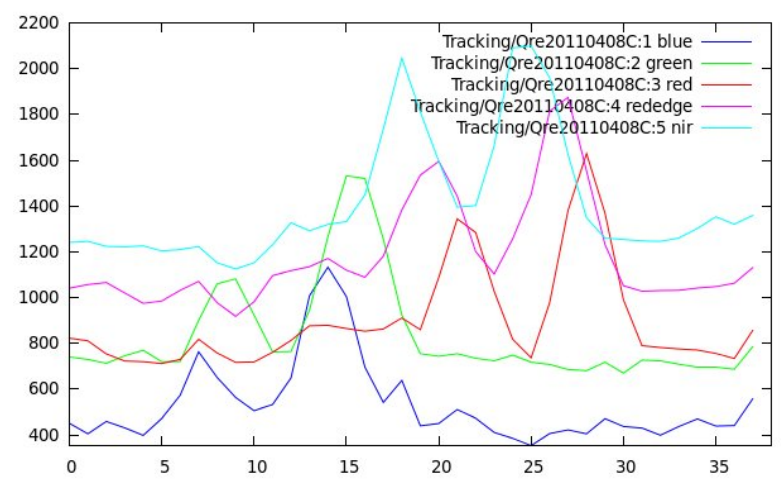

Figure 22: Profile along green line of RapidEye scene in fig. 21, reflections $\times 100$ in [\%] vs. metres along road

An assessment was made along a $15 \mathrm{~km}$ long strip of the A96. The results are shown in tab. 6 . Along the highway 61 vehicles were manually detected, 81 were detected by the presented automatic method. From these 46 where found erroneously (mostly in clouds) and 21 manually marked objects were missed - mostly due to the too bad contrast. 21 objects were detected correctly whereas 14 detected objects were detected but correlated with the wrong mate in the other band. Typical errors can be seen in fig. 23.

Table 6: Accuracy assessment of detected objects along a $15 \mathrm{~km}$ strip of the A96

\begin{tabular}{|l|l|}
\hline 81 & Detected in total \\
61 & Reference \\
21 & Detected correctly \\
14 & Detected, but correlated wrong \\
26 & Not detected (mostly too bad contrast) \\
46 & Detected erroneous (mostly clouds) \\
\hline
\end{tabular}

\subsection{Pléiades}

For assessing our algorithm with the Pléiades sensor we used a scene acquired 2012-02-25 over Melbourne, Australia. As shown in fig. 24 we evaluated the quality of our method on a $4 \times 2 \mathrm{~km}^{2}$ section of the harbour of Melbourne containing a strip of the M1 highway. Fig. 24 shows the 265 manually measured cars (and one ship, yellow crosses) together with all 300 automatically found objects (green crosses).

Applying the method to the Melbourne-harbour-image finds 300 moving objects. As shown in tab. 7 the quality is not so good. Only 115 of 265 objects were detected correctly which corresponds to a detection rate of only $43.4 \%$. In contrast 185 of the 300 detected objects were no moving objects (false detect rate of $61.7 \%)$.

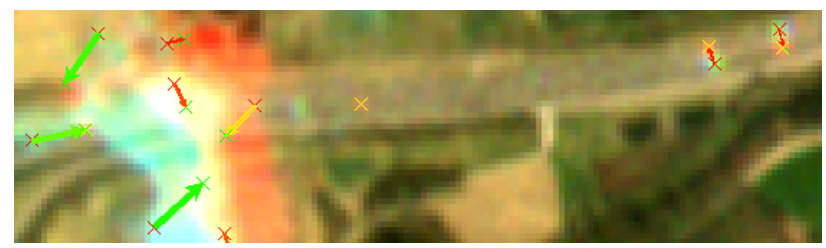

Figure 23: Typical errors in a RapidEye Scene, left on border: correctly detected car at cloud-border, left: erroneous detections in clouds, center: (single yellow cross) missed car due to bad contrast, right: wrong correlation of cars on opposite lanes

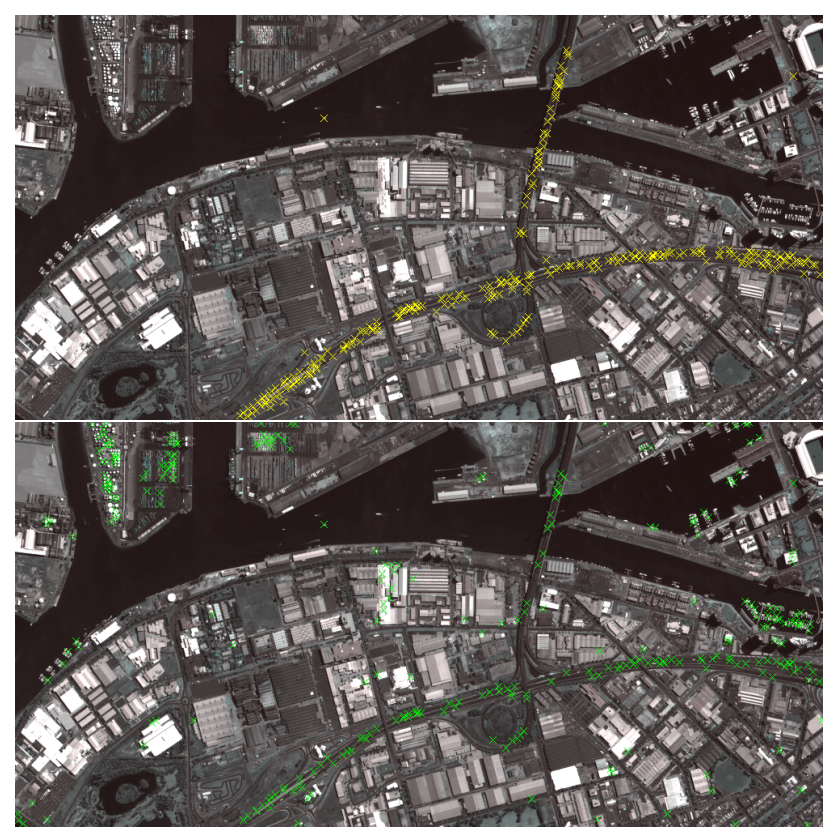

Figure 24: Example Pléiades image, $4 \times 2 \mathrm{~km}^{2}$, harbour of Melbourne (Australia), top: reference of manually measured cars (yellow crosses), bottom: automatically found moving objects (green crosses)

As can be seen in fig. 24 these erroneously detected objects are located mostly in the top left of the scene where the oil terminal with many oiltanks and in the marina on the right center containing many small ships. However also one ship (upper part, left of center) was found by the method even if the speed was absolutely overestimated with $139 \mathrm{~km} / \mathrm{h}$ instead of $0.8 \mathrm{~m} / 0.16 \mathrm{~s}$ or $18 \mathrm{~km} / \mathrm{h}$.

\subsection{SkyBox}

For assessing the SkyBox system an image from 2014-08-09 in the south of France near Fos-sur-Mer (Camargue, France) was available. In the orthorectified scene 8 of detector $2\left(3 \times 2 \mathrm{~km}^{2}\right)$ all moving objects were marked manually and automatically detected in 11 seconds as shown in fig. 25.

Assessment of the result vs. the manually measurement shows 21 automatically detected objects and 22 manually measured objects. From these 12 were correct detected, 10 cars were missed and 9 objects - mostly in the industrial area in the bottom center of the image - were wrongly detected as moving object. So for SkyBox using the red and green band also a detection rate of $54.5 \%$ and a false-detect-rate of $42.9 \%$ can be found while no miscorrelations were found in the test scene.

\section{RESULTS}

In tab. 8 all results from the above experiments are summarized. The detection rate is defined as correctly detected cars divided 
Table 7: Pléiades accuracy assessment

\begin{tabular}{|r|l|}
\hline 300 & Detected in total \\
265 & Reference \\
111 & Detected correctly \\
185 & Detected erroneous \\
4 & Correlated wrong \\
\hline
\end{tabular}
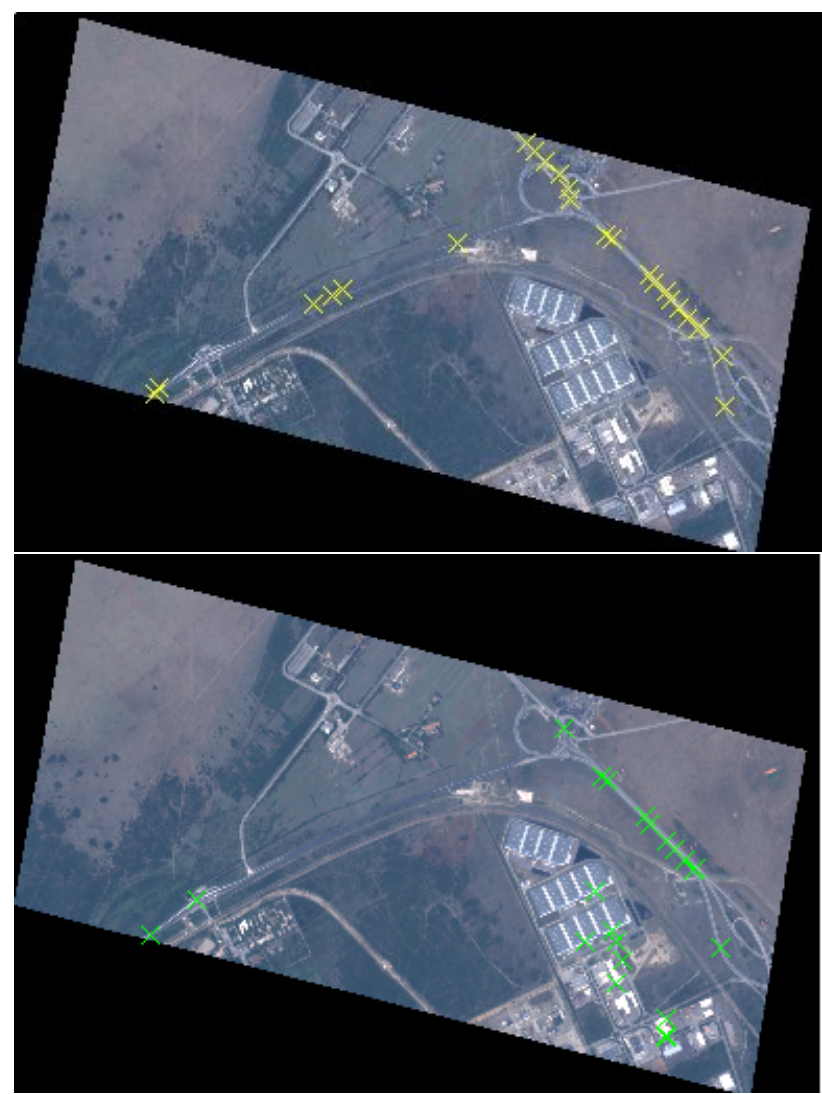

Figure 25: Example SkyBox image, $3 \times 2 \mathrm{~km}^{2}$ near Fos-surMer in southern France, top: reference of manually measured cars (yellow crosses), bottom: automatically found moving objects (green crosses)

by all manually measured cars. The false-detect-rate is the number of objects detected automatically but not manually verified divided by all automatically detected objects. The miscorrelation rate is the number of wrongly correlated objects divided by the number of all correctly detected objects (so the object was found correctly in one band, but the wrong mate in the other band was taken for the speed calculation).

Table 8: Quality measures for the assessed sensors

\begin{tabular}{|l|r|r|r|}
\hline Sensor & Detection rate & False-detect-rate & Miscorrelation-rate \\
\hline World-View-2 & $51.3 \%$ & $42.9 \%$ & $20.9 \%$ \\
RapidEye & $57.4 \%$ & $56.8 \%$ & $40.0 \%$ \\
Pléiades & $43.4 \%$ & $61.7 \%$ & $3.5 \%$ \\
SkyBox & $54.5 \%$ & $42.9 \%$ & $0.0 \%$ \\
\hline
\end{tabular}

The missed objects (objects not detected, $100 \%$ - "detection rate") are mostly due to too low contrast of the object relative to the road. So cars with the same color as the road cannot be detected. Also cars darker than the road are detected not so well. If a dark car with good contrast to the road is detected the presented method will give the wrong driving direction - but this happened only by investigating the WorldView-2 image and only in $5.6 \%$ of all detected cars ("reversed direction" in tab. 5: 50/894). In the RapidEye image e.g. the image quality was too bad to detect dark cars on the road.

The false-detect-rate mostly contains objects far away from streets with spectral signatures which look similar to those of moving objects. This rate may be reduced dramatically by introducing a road mask.

The miscorrelation is mostly based on better and nearer matches on neighbouring lanes as shown in fig. 23 (right). So this may be reduced also using the road mask and only allowing correlations along road directions.

With this presented method the Pléiades sensor perfom badest due to the very low time gap of only $0.16 \mathrm{~s}$ which results in large overlaps of also small moving objects. Consequently the derived speeds are mostly too high (even for cars!) if the objects have a remaining overlap in the two investigated bands. Additionally the channel merging method proposed for Pléiades in eqs. 1 and 2 give some artifacts on borders of buildings, ships and - as seen in the test area - oiltanks which result in a huge number of erroneously detected objects.

The processing time was for a complete WorldView- 2 scene $(16 \times$ $20 \mathrm{~km}^{2}$ at a GSD of $2 \mathrm{~m}$ ) only about 2.5 minutes on a standard Linux-PC (8 core (only 1 used), $2.5 \mathrm{GHz}, 24 \mathrm{~GB}$ RAM). The full $20 \times 12 \mathrm{~km}^{2}$ Pléiades scene needs 5 minutes due to the huge amount of 550000 object candidates and only 5000 remaining correlated objects.

\section{CONCLUSION AND OUTLOOK}

We presented in this paper the most simple possible method for automatic detection of moving objects from only single very high resolution (VHR) satellite scenes covering the whole area. The method utilizes a common feature for most VHR satellite sensors where CCD sensor elements are mounted with a recognizable distance on the focal plane array (FPA) of the sensor.

This feature results in acquisition of moving objects at different positions in these different CCD elements. The presented method finds moving objects in different acquired bands, correlates them and calculates the speed of the objects by applying the previously derived time-gap between the acquisition of the bands.

This simplest-as-possible method already gives good results. Even with a relatively low resolution sensor like RapidEye with a nominal ground sampling distance (GSD) of only $6.5 \mathrm{~m}$ moving cars can be detected and measured.

The assessed detection rate for the four investigated sensors WorldView-2, RapidEye, Pléiades and SkyBox - is always about $50 \%$. But also the false-detect-rate is about $50 \%$. In all cases large trucks give wrong speed results using this method. Similarly dark cars on bright roads give the reversed direction.

For future extensions of the method first not only the diffence images should be used for speed extraction but only for object detection. The speed extraction should be done in a separate step by re-mapping the detected objects to the original bands and correlating the whole detected objects from these bands with each other. A second refinement of the method would be of course the usage of a road layer. So only objects on roads will be taken into account. As shown in the results above in this way most of all false detected moving objects will be removed. Also a road layer will allow to reduce the miscorrelation of objects on neighbouring lanes by allowing only correlations in road direction. But as can be seen in the RapidEye images additionally to the road-layer a cloud-mask has to be used. 
In summary it can be concluded that the (refined) method is suitable for acquiring a large area traffic situation from only one single satellite image of many different sensors in a short time.

\section{REFERENCES}

Delvit, J.-M., Greslou, D., Amberg, V., Dechoz, C., Delussy, F., Lebegue, L., Latry, C., Artigues, S. and Bernard, L., 2012. Attitude Assessment using Pleiades-HR Capabilities. In: International Archives of the Photogrammetry, Remote Sensing and Spatial Information Sciences, Vol. 39 B1, pp. 525-530.

Etaya, M., Sakata, T., Shimoda, H. and Matsumae, Y., 2004. An Experiment on Detecting Moving Objects Using a Single Scene of QuickBird Data. Journal of the Remote Sensing Society of Japan 24(4), pp. 357-366.

Kääb, A., 2011. Vehicle velocity from WorldView-2 satellite imagery. In: IEEE Data Fusion Contest, Vol. 2011.

Krauß, T., Stätter, R., Philipp, R. and Bräuninger, S., 2013. Traffic Flow Estimation from Single Satellite Images. ISPRS Archives XL-1/W, pp. 241-246.

Leitloff, J., 2011. Detektion von Fahrzeugen in optischen Satellitenbildern. PhD thesis, Technische Universität München.

M. Pesaresi, K. Gutjahr, E. P., 2007. Moving Targets Velocity and Direction Estimation by Using a Single Optical VHR Satellite Imagery. In: International Archives of Photogrammetry, Remote Sensing and Spatial Information Sciences, Vol. 36 3/W49B, pp. 125-129.

RapidEye, 2012. Satellite Imagery Product Specifications. Technical report, RapidEye.

Tao, J. and Yu, W.-x., 2011. A Preliminary study on imaging time difference among bands of WorldView- 2 and its potential applications. In: Geoscience and Remote Sensing Symposium (IGARSS), 2011 IEEE International, Vol. 2011, pp. 198-200.

\section{ACKNOWLEDGEMENTS}

We would like to thank Sarah Bräuninger and Robert Philipp for conducting the huge amount of tedious manually measurements and preparing many images and diagrams for this work. Furthermore we thank Astrium for providing a multi-stereo Pléiades scene from Melbourne in the scope of the "Pléiades User Group" initiative. 